Aim of the study: Hepatocellular carcinoma (HCC) is common throughout the world. Most HCCs are diagnosed at an advanced stage. There is an urgent need to find new methods for screening and surveillance of individuals at risk for HCC. The aim of this study was to evaluate serum $\alpha$-fetoprotein (AFP)-L3 and serum Golgi protein 73 (GP73) detection in diagnosis of HCC with different AFP concentration.

Material and methods: One hundred and eighty one patients were involved, including 102 with HCC and 79 with benign liver disease. The serum AFP-L3 and GP73 was measured by a liquid-phase binding assay and quantitative enzyme-linked immunosorbent assay, respectively.

Results: Of the 102 HCC patients, 53 were positive for AFP, 77 were positive for AFP-L3, and 79 were positive for GP73. The maximum area under the curve for AFP-L3\% and for GP73 was significantly different from the AUC of 0.5525 for total AFP $(p<0.01)$. AFP-L3\% was not detected for AFP $<20 \mathrm{ng} / \mathrm{ml}$ However, elevated GP73 was detected in $87.50 \%$ of the patients. In the HCC patients with total AFP $20-400 \mathrm{ng} / \mathrm{ml}$, elevated AFP-L3 was detected in $26 \mathrm{pa}$ tients, whereas in 23 patients elevated GP73 could be detected. In the HCC patients with a total AFP > $400 \mathrm{ng} / \mathrm{ml}$, AFP-L3\% > 10\% was present in $96.23 \%$, and GP73 was detected in $87.50 \%$.

Conclusions: The determination of AFP-L3\% and GP73 in combination with AFP can increase the sensitivity and specificity in diagnosis of HCC $\alpha$-fetoprotein-L3\% and GP73, in combination with AFP, are useful biomarkers to confirm the diagnosis of HCC.

Key words: $\alpha$-fetoprotein, lectin, reactive $\alpha$, fetoprotein, hepatocellular carcinoma.

Contemp Oncol (Pozn) 2014; 18 (3): 192-196 DOI: $10.5114 /$ wo.2014.43157

\section{Evaluation of $\alpha$-fetoprotein-L3 and Golgi protein 73 detection in diagnosis of hepatocellular carcinoma}

\author{
Zhiling Jia, Li Wang, Chang Liu, Zhonghe Yu, Lina Chai, Mancang Zhao
}

Department of Oncology, General Hospital of Beijing Command of PLA, Beijing, PR China

\section{Introduction}

Hepatocellular carcinoma (HCC) is common throughout the world, and represents the fifth most common cancer in the world and the third most frequent cause of mortality amongst oncological patients [1]. It is responsible for more than 500000 deaths with over 600000 new cases yearly worldwide [2]. The high mortality associated with HCC is partly due to unresponsiveness to treatment, with a 5-year survival rate of $<5 \%$ after diagnosis [3]. When diagnosed at an early stage, treatment of HCC with surgical resection or liver transplantation can be curative. In fact, most HCCs are diagnosed at an advanced stage. Therefore, there is an urgent need for improved methods of screening and surveillance of individuals at risk for HCC.

$\alpha$-fetoprotein (AFP) has served as a diagnostic test for HCC since the 1970s, due to there being a correlation between elevated levels of AFP and the occurrence of HCC. However, AFP as a sole indicator of HCC is of limited value. The diagnostic sensitivity of AFP for small HCCs is relatively low. Furthermore, AFP levels are elevated both in patients with HCC and in those with chronic liver diseases, and there is a wide overlap between the two groups. $\alpha$-fetoprotein is often elevated in patients with chronic hepatitis $\mathrm{C}$ virus infection in the absence of HCC and not elevated in as many as 50\% of HCC [4]. Although it has relatively poor sensitivity and specificity as a surveillance test, serum AFP, used in combination with serial liver ultrasound examination, is a standard means for risk stratification, screening and surveillance for HCC.

The chemical structure of AFP shows that different sugar moieties of the bonds determine their binding capacity to lectin Lens culinaris agglutinin (LCA) [5]. $\alpha$-fetoprotein- L3, as the LCA-bound fraction, is the major glycoform in the serum of HCC patients [6-8]. The AFP-L3 isoform has been reported to be more specific for the diagnosis of HCC than total AFP. It has also been shown to be associated with more aggressive HCCs, and to predict a worse outcome [9]. Recent reports also suggest that it is useful for predicting the risk of development of HCC in patients with chronic liver disease.

Another useful biomarker for HCC is Golgi protein 73 (GP73). Golgi protein 73 (also called GOLPH2) is a Golgi type II transmembrane protein of unknown function that is constitutively expressed in biliary epithelial cells in normal livers. It has been reported that GP73 is up-regulated at high levels in hepatocytes of patients with acute and chronic hepatitis and HCC [10-12]. Golgi protein 73 is highly expressed in hepatocytes of patients with HCC, and secreted into the serum of HCC patients following furin-mediated proteolytic cleavage of its $N$-terminus [13], a feature that can be exploited for the diagnosis of HCC [14]. Although its function has yet to be elucidated, its expression profile suggests that GP73 may serve as a biomarker of HCC. 
In this study, we detected the serum AFP, AFP-L3\% and GP73 in patients with HCC and benign liver diseases and compared their diagnostic utility in HCC.

\section{Material and methods}

\section{Patients}

We collected serum samples from 181 adult patients (158 men and 23 women, age ranged from 25 to 78 years, median age 52 years) evaluated for HCC or chronic liver disease between September 2009 and July 2010. The samples were stored at $-70^{\circ} \mathrm{C}$.

Of the 181 patients, 102 had HCC and 79 had benign liver disease. One hundred serum samples were from healthy volunteers (71 men and 29 women, age ranged from 25 to 60 years, median age 50 years). This study was conducted in accordance with the Declaration of Helsinki. This study was conducted with approval from the Ethics Committee of the General Hospital of Beijing Command of PLA. The patients of this series were all from the General Hospital of Beijing Military Area Command of PLA. Written informed consent was obtained from all participants.

Cases were selected for whom a specimen was available that had been drawn after diagnosis of HCC but before any therapy. The diagnosis of HCC was based on either histopathology or on non-invasive criteria. The non-invasive diagnosis of HCC was established based on the following criteria revised by the Society of Liver Cancer of the Chinese Anti-Cancer Association in September, 2001.

1) Total AFP $\geq 400 \mu g / l$, excluding pregnancy, germinal and embryonal neoplasm, active liver disease and metastatic liver cancer, rigid liver mass could be palpated, or typical occupying lesion of HCC ascertained by imaging modalities (including ultrasound, contrast CT, contrast $\mathrm{MRI}$, and angiography);

2) Total AFP $<400 \mu \mathrm{g} / \mathrm{l}$, excluding pregnancy, germinal and embryonal neoplasm, active liver disease and metastatic liver cancer, typical occupying lesion of HCC ascertained by two imaging modalities (including contrast CT, contrast $\mathrm{MRI}$, and angiography) or two HCC biomarkers (including DCP, GGT II, AFU and CA19-9) elevated, and typical occupying lesion of HCC ascertained by one imaging modality (including contrast CT, contrast MRI, and angiography).

3) Clinical manifestation in accordance with HCC and confirmed metastatic tumor except liver mass (including bloody pyoperitoneum or tumor cells found in pyoperitoneum), excluding metastatic liver cancer.

Clinical and laboratory information at the time of diagnosis were retrospectively ascertained from the medical records. Laboratory findings abstracted included liver biochemistries (AST, ALT, alkaline phosphatase, total and direct bilirubin, albumin), platelet count, prothrombin time, creatinine, hepatitis B and C serologies. Histopathology slides for patients who underwent surgical resection or needle biopsy were reviewed by a liver pathologist.

\section{Detection of serum $\alpha$-fetoprotein and $\alpha$-fetoprotein-L3}

Total serum AFP levels were determined by enzyme immunoassay using commercially available kits (ABBOTT
AXSYM). AFP-L3 was measured by a liquid-phase binding assay. Briefly, AFP-L3 in the samples was isolated via lectin-affinity adsorption using the tube coupled with antibody-affinity blotting (Hotgen Co., Ltd.), after elution, and then quantified using chemiluminescence on the automated platform as AFP. The ratio of LCA-reactive AFP to total AFP (AFP-L3\%) was calculated. The typical inter-assay variance for this test, expressed as the coefficient of variance, is between $2.8 \%$ and $13.4 \%$ for AFP-L3\% and $2.6 \%$ and $4.6 \%$ for AFP concentration. The lower limit of detection for total AFP concentration was $0.8 \mathrm{ng} / \mathrm{ml}$ and for AFP-L3\% 0.5\%. If AFP-L3 was detected in the sample, the system only provided a reliable value of AFP-L3\% for samples when the total AFP concentration was $10 \mathrm{ng} / \mathrm{ml}$. For samples with total AFP of $>300,000 \mathrm{ng} / \mathrm{ml}$, we elected not to assess AFP-L3\% because of the need for multiple sequential dilutions of the sample. The recommended cutoff points for AFP and AFP-L3 were $400 \mathrm{ng} / \mathrm{ml}$ and 10\%, respectively.

\section{Detection of serum Golgi protein 73}

The serum concentration of Golgi protein 73 (GP73) was detected by a quantitative enzyme-linked immunosorbent assay kit (Hotgen Co., Ltd.). The standard substance of GP73 with concentration of $50 \mathrm{ng} / \mathrm{ml}, 120 \mathrm{ng} /$ $\mathrm{ml}, 300 \mathrm{ng} / \mathrm{ml}$ and $500 \mathrm{ng} / \mathrm{ml}$ was measured, and a standard curve was produced graphically. The optical density (OD) of the samples was compared to the standard curve. For the measured quantity (a given Y-axis value), the substance concentration (X-axis value) could be calculated correspondingly. The cut-off point of GP73 was $150 \mathrm{ng} / \mathrm{ml}$.

\section{Statistical analysis}

For correlations between tumor markers, Pearson's correlation coefficient was used, with values of -0.4 to 0.4 considered as having no correlation. Data for marker concentrations are reported as the median with $25^{\text {th }}$ and $75^{\text {th }}$ percentiles.

Comparison of continuous and dichotomous variables between the two groups was performed using the Mann-Whitney U-test, the $\chi^{2}$ test, or Fisher's exact test. The statistical analysis was performed using the SPSS13.0 software package. To compare abilities of tumor markers in diagnosis of HCC, receiver operator characteristic (ROC) curves, which correlate true- and false-positive rates [sensitivity and (1-specificity)], were constructed using the ROCKIT program. Additionally, areas under the ROC curve (AUC) were calculated for each marker. The statistical significance of differences between the two AUCs also was determined.

\section{Results}

Detection of serum $\alpha$-fetoprotein and $\alpha$-fetoprotein-L3 and Golgi protein 73

The comparative analysis of AFP, AFP-L3 and GP73 in patients with different liver diseases and healthy persons is shown in Table 1. The recommended cut-off points for 
Table 1. Comparison of AFP, AFP-L3 and GP73 detection in patients with HCC

\begin{tabular}{|c|c|c|c|c|}
\hline Group & Cases & AFP positive (\%) & AFP-L3 positive (\%) & GP73 positive (\%) \\
\hline $\mathrm{HCC}$ & 102 & $53(51.96)$ & $77(75.50)$ & $79(77.45)$ \\
\hline benign liver diseases & 79 & 39 (49.37) & $5(6.33)$ & $20(25.32)$ \\
\hline healthy persons & 100 & 0 & 0 & 0 \\
\hline
\end{tabular}

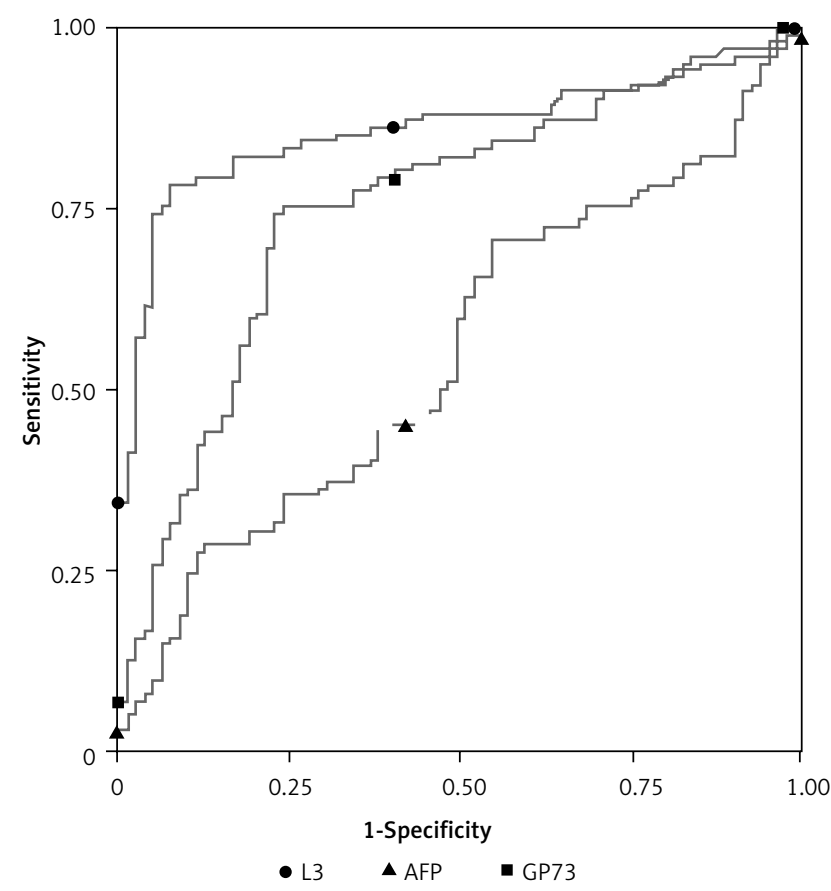

Fig. 1. ROC curves for the diagnosis of HCC using total AFP, AFP-L3\% and GP73

Table 2. Comparison of AFP-L3 and GP73 in HCC patients with different AFP concentrations

\begin{tabular}{lccc} 
AFP $(\mathrm{ng} / \mathrm{ml})$ & Cases & $\begin{array}{c}\text { AFP-L3 } \\
\text { positive (\%) }\end{array}$ & $\begin{array}{c}\text { GP73 } \\
\text { positive (\%) }\end{array}$ \\
\hline $0-20$ & 16 & $0(0)$ & $14(87.50)$ \\
$20-400$ & 33 & $26(78.79)$ & $23(69.70)$ \\
$\geq 400$ & 53 & $51(96.23)$ & $40(75.47)$
\end{tabular}

AFP, AFP-L3 and GP73 were $400 \mathrm{ng} / \mathrm{ml}, 10 \%$, and $150 \mathrm{ng} /$ $\mathrm{ml}$ respectively. The AFP, AFP-L3 and GP73 levels were significantly elevated in the HCC group over the benign liver disease and healthy persons groups. Of the 102 samples from patients with hepatocellular carcinoma, 53 samples (51.96\%) were positive for AFP, 77 samples (75.50\%) were positive for AFP-L3, and 79 samples $(77.45 \%)$ were positive for GP73. There were significant differences in the rate between AFP and AFP-L3, AFP and GP73.

\section{Diagnostic utility of $\alpha$-fetoprotein and $\alpha$-fetoprotein-L3\% and Golgi protein 73}

Receiver operator characteristic curves for the diagnosis of HCC using total AFP, AFP-L3\% and GP73 are shown in Fig. 1. The maximum area under the curve (AUC) for distinguishing between HCC and benign liver disease was 0.8625 for AFP-L3\% and 0.7538 for GP73, almost significantly different from the AUC of 0.5525 for total AFP ( $p<0.01$ ). With the recommended AFP-L3\% cut-off point of $=10 \%$, the sensitivity with GP73 cut-point of $=150 \mathrm{ng} /$ $\mathrm{ml}$, the sensitivity of the GP73 for HCC was $71 \%$ and the specificity was $63 \%$ (Table 2 ).

Comparison of $\alpha$-fetoprotein-L3 and Golgi protein 73 detection in hepatocellular carcinoma patients with different $\alpha$-fetoprotein concentrations

In all patients, a small majority of HCC patients had a total AFP < 20 ng/ml (16/102; 15.69\%), 32.35\% (33/101) had a total AFP $20-400 \mathrm{ng} / \mathrm{ml}$, and 51.96\% (53/102) had a total AFP > $400 \mathrm{ng} / \mathrm{ml}$.

Since AFP-L3\% is typically not detected for AFP $<20 \mathrm{ng} /$ $\mathrm{ml}$, AFP-L3\% was not relevant for the diagnosis of HCC for individuals with a total AFP $<20 \mathrm{ng} / \mathrm{ml}$. However, an elevated level of GP73 can be detected in $87.50 \%$ (14/16) of the patients in this subset, and the maximum area under the curve (AUC) for distinguishing between HCC and benign liver disease was 0.9766 for GP73 (Fig. 2A).

Twenty-six $(78.79 \%)$ of the HCC patients with total AFP $20-400 \mathrm{ng} / \mathrm{ml}$ showed an elevated level of AFP-L3, whereas $23(69.70 \%)$ of the HCC patients in this subset showed an elevated level of GP73. The AUC for distinguishing between HCC and benign liver disease was 0.9063 for AFP-L3\% and 0.7803 for GP73 (Fig. 2B).

On the other hand, AFP-L3\% >10\% was present in 96.23\% (51/53) of the patients with HCC and for total AFP $>400 \mathrm{ng} / \mathrm{ml}$, GP73 could be detected in $87.50 \%(14 / 16)$ of the patients in this subset. The AUC for distinguishing between HCC and benign liver disease was 0.9690 for AFP-L3\% and 0.6425 for GP73 (Fig. 2C).

\section{Discussion}

High mortality rates for HCC have significantly increased worldwide. Regular follow-up of chronic liver disease patients is indispensable for early detection of HCC $[15,16]$. Serum AFP is the most widely used biomarker for diagnosis of HCC. The normal range for serum AFP levels is $10-20 \mathrm{ng} / \mathrm{ml}$ and a level $>400 \mathrm{ng} / \mathrm{ml}$ is usually regarded as of diagnostic value. However, two thirds of HCC patients with the nodule less than $4 \mathrm{~cm}$ have serum AFP levels less than $200 \mathrm{ng} / \mathrm{ml}$ and up to $20 \%$ of HCC patients do not produce AFP. Mild elevation of AFP also occurs in viral hepatitis and other liver diseases $[17,18]$. It has limited utility of differentiating HCC from benign hepatic disorders. 
A

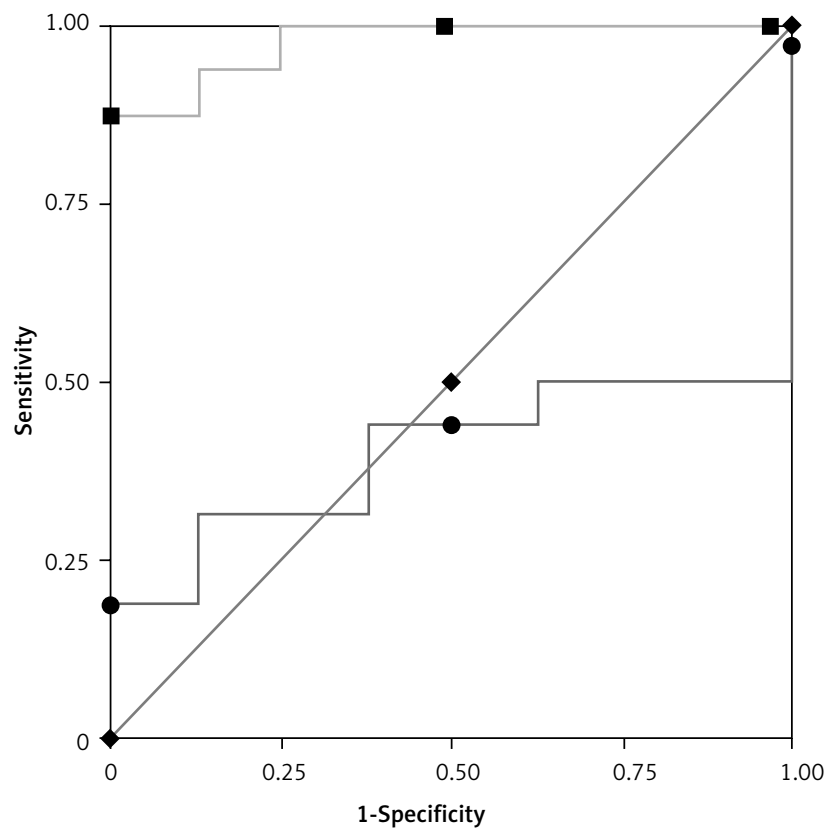

C

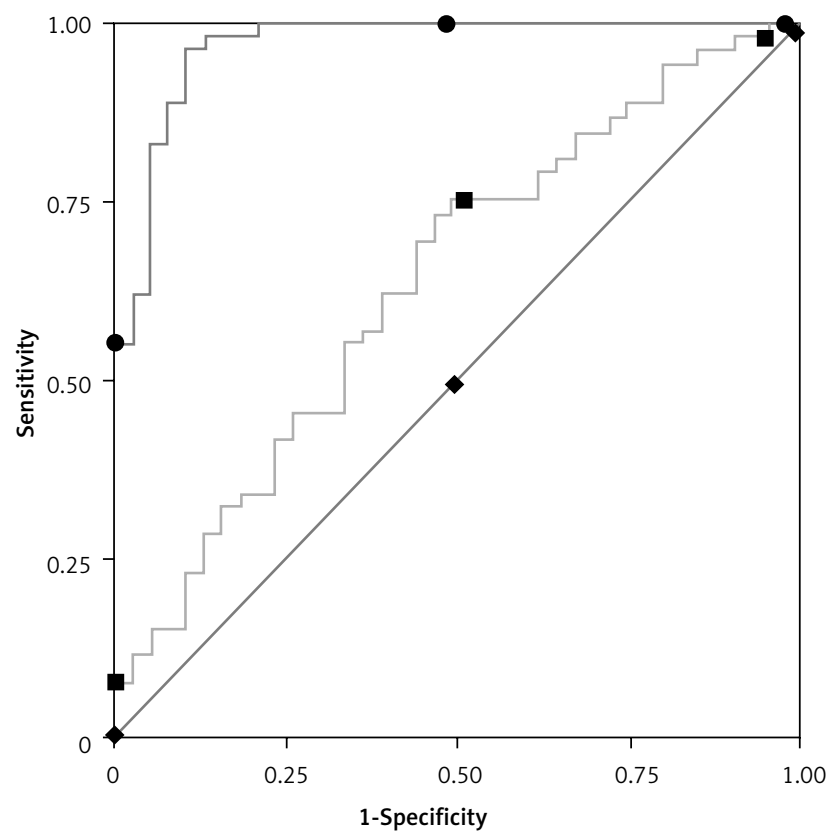

B

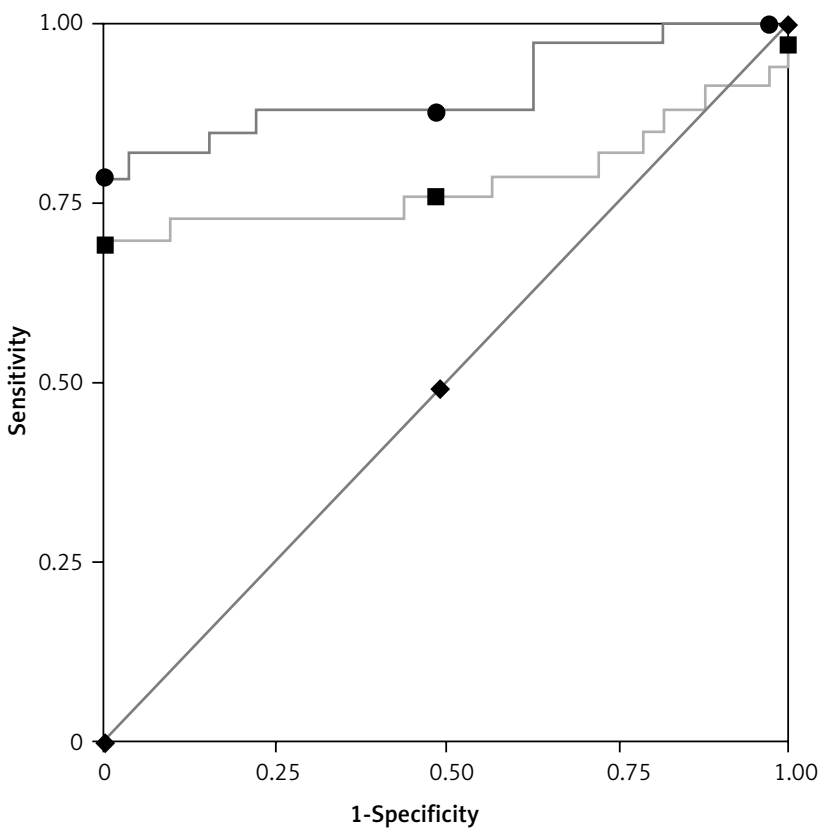

$\alpha$-fetoprotein-L3, as the LCA-bound fraction in total AFP, is also widely used as a tumor marker for HCC, and has been shown to be more valuable than AFP in differentiating HCC from nonmalignant hepatopathy, detecting small HCC, and predicting the prognosis [19]. In this study, with the recommended cut-off point of AFP-L3\% $=10$, the sensitivity of AFP-L3\% for HCC was $71 \%$ and the specificity was $63 \%$, significantly higher than total AFP. Since AFP-L3\% is typically not reported for an AFP $<20 \mathrm{ng} / \mathrm{ml}$, AFP-L3\% was not relevant for the diagnosis of HCC for individuals with a total AFP $<20 \mathrm{ng} / \mathrm{ml}$. On the other hand, the patients with a total AFP $>400 \mathrm{ng} / \mathrm{ml}$ and typical liver mass could be diagnosed with HCC; therefore the AFP-L3\% again provided little benefit for the diagnosis of HCC in these pa- tients. However, the patients with total AFP in the intermediate $20-400 \mathrm{ng} / \mathrm{ml}$ range, frequently individuals with chronic hepatitis B or C virus infection, often present a diagnostic dilemma, as they often have false-positive elevations of total AFP. For the total AFP range of 10-10,000 ng/ $\mathrm{ml}$, the AUC for distinguishing between HCC and benign liver disease was 0.80 for AFP-L3\% and 0.78 for total AFP $(p=0.754)$. For the total AFP range of $10-400 \mathrm{ng} / \mathrm{ml}$, the AUC for diagnosis of HCC using AFP-L3\% was 0.76, almost significantly different from the AUC of 0.59 for total AFP $(p=0.074)$. $\alpha$-fetoprotein- $L 3 \%>40$ is seldom observed in patients with benign liver disease. Thus, AFP-L3\% is more valuable in differentiating HCC and benign liver disease in patients with a total AFP range of 10-200 ng/ml. 
Golgi protein 73 is a newly introduced biomarker for HCC. The correlation between serum GP73 levels and a diagnosis of HCC in people has been confirmed recently. Total GP73 levels were shown to have a positive predictive value equal to or greater than AFP $[20,21]$. In this study, with the recommended cut-off point of GP73 $=150 \mathrm{ng} / \mathrm{ml}$, the sensitivity of GP73 for HCC was $71 \%$ and the specificity was $63 \%$, significantly higher than total AFP. For diagnostic utility in all patients, GP73 > AFP-L3 > AFP in sensitivity, and AFP-L3 > GP73 > AFP in specificity.

In this study, a small majority of HCC patients (63/166; $38 \%$ ) had a total AFP $<20 \mathrm{ng} / \mathrm{ml}$, whereas $56 \%$ of benign liver disease patients had a total AFP $<20 \mathrm{ng} / \mathrm{ml}$. It is reported that AFP is not elevated in as many as $50 \%$ of HCC cases, especially in its early stage [8]. Because AFP-L3\% is negative in these patients, AFP-L3 detection could not help to distinguish HCC from benign liver disease in this group. However, serum GP73 elevated in \% HCC patients with a total AFP $<20 \mathrm{ng} / \mathrm{ml}$, elevated in \% benign liver disease patients. For the total AFP range of $10-100 \mathrm{ng} / \mathrm{ml}$, the AUC for diagnosis of HCC using AFP-L3\% was 0.76 , almost significantly different from the AUC of 0.59 for GP73. Golgi protein 73 is more useful for distinguishing between HCC and benign liver disease in patients with a total AFP range of $10-100 \mathrm{ng} / \mathrm{ml}$, especially total AFP $<20 \mathrm{ng} / \mathrm{ml}$.

In conclusion, our data suggest that the determination of AFP-L3\% and GP73 in combination with AFP increases the sensitivity and specificity in diagnosis of HCC, especially in individuals with total AFP $<400 \mathrm{ng} / \mathrm{ml}$. Thus, AFP-L3\% and GP73 should be useful biomarkers, in combination with AFP, to confirm the diagnosis of HCC.

The authors declare no conflict of interest.

\section{References}

1. Bosch FX, Ribes J, Cléries R, Díaz M. Epidemiology of hepatocellular carcinoma. Clin Liver Dis 2005; 9: 191-211.

2. Parkin DM, Bray F, Ferlay J, Pisani P. Global cancer statistics, 2002. CA Cancer J Clin 2005; 55: 74-108.

3. El-Serag HB, Mason AC, Key C. Trends in survival of patients with hepatocellular carcinoma between 1977 and 1996 in the United States. Hepatology 2001; 33: 62-5.

4. Block TM, Mehta AS, Fimmel CJ, Jordan R. Molecular viral oncology of hepatocellular carcinoma. Oncogene 2003; 22: 5093-107.

5. Zhou L, Liu J, Luo F. Serum tumor markers for detection of hepatocellular carcinoma. World J Gastroenterol 2006; 12: 1175-81.

6. Hayashi K, Kumada T, Nakano S, et al. Usefulness of measurement of Lens culinaris agglutinin-reactive fraction of alphafetoprotein as a marker of prognosis and recurrence of small hepatocellular carcinoma. Am I Gastroenterol 1999; 94: 3028-33.

7. Okuda K, Tanaka M, Kanazawa N, et al. Evaluation of curability and prediction of prognosis after surgical treatment for hepatocellular carcinoma by lens culinaris agglutinin-reactive alpha-fetoprotein. Int J Oncol 1999; 14: 265-71.

8. Malaguarnera G, Giordano M, Paladina I, Berretta M, Cappellani A, Malaguarnera M. Serum markers of hepatocellular carcinoma. Dig Dis Sci 2010; 55: 2744-55.

9. Song BC, Suh DJ, Yang SH, Lee HC, Chung YH, Sung KB, Lee YS. Lens culinaris agglutinin-reactive alpha-fetoprotein as a prognostic marker in patients with hepatocellular carcinoma undergoing transcatheter arterial chemoembolization. I Clin Gastroenterol 2002; 35: 398-402.
10. Kladney RD, Bulla GA, Guo L, Mason AL, Tollefson AE, Simon DJ, Koutoubi Z, Fimmel CJ. GP73, a novel Golgi localized protein upregulated by viral infection. Gene 2000; 249: 53-65.

11. Kladney RD, Cui X, Bulla GA, Brunt EM, Fimmel CJ. Expression of GP73, a resident Golgi membrane protein, in viral and nonviral liver disease. Hepatology 2002; 35: 1431-40.

12. Iftikhar R, Kladney RD, Havlioglu N, et al. Disease- and cell-specific expression of GP73 in human liver disease. Am J Gastroenterol 2004; 99: 1087-95.

13. Bachert C, Fimmel C, Linstedt AD. Endosomal trafficking and proprotein convertase cleavage of cis Golgi protein GP73 produces marker for hepatocellular carcinoma. Traffic 2007; 8: 1415-23.

14. Marrero JA, Romano PR, Nikolaeva O, et al. GP73, a resident Golgi glycoprotein, is a novel serum marker for hepatocellular carcinoma. J Hepatol 2005; 43: 1007-12.

15. Sherman M, Peltekian KM, Lee C. Screening for hepatocellular carcinoma in chronic carriers of hepatitis B virus: incidence and prevalence of hepatocellular carcinoma in a North American urban population. Hepatology 1995; 22: 432-8.

16. Shimauchi Y, Tanaka M, Kuromatsu R, et al. A simultaneous monitoring of Lens culinaris agglutinin A-reactive alpha-fetoprotein and des-gamma-carboxy prothrombin as an early diagnosis of hepatocellular carcinoma in the follow-up of cirrhotic patients. Oncol Rep 2000; 7: 249-56.

17. Sato Y, Nakata K, Kato Y, et al. Early recognition of hepatocellular carcinoma based on altered profiles of alpha-fetoprotein. N Engl J Med 1993; 328: 1802-6.

18. Hu KQ, Kyulo NL, Lim N, Elhazin B, Hillebrand DJ, Bock T. Clinical significance of elevated alphafetoprotein (AFP) in patients with chronic hepatitis C, but not hepatocellular carcinoma. Am J Gastroenterol 2004; 99: 860-5.

19. Li D, Mallory T, Satomura S. AFP-L3: a new generation of tumor marker for hepatocellular carcinoma. Clin Chim Acta 2001; 313: 15-9.

20. Ozkan H, Erdal H, Tutkak H, Karaeren Z, Yakut M, Yüksel O, Köklü S. Diagnostic and prognostic validity of golgi protein 73 in hepatocellular carcinoma. Digestion 2011; 83: 83-8.

21. Mao Y, Yang H, Xu H, et al. Golgi protein $73(\mathrm{GOLPH} 2)$ is a valuable serum marker for hepatocellular carcinoma. Gut 2010; 59: 1687-93.

\section{Address for correspondence}

\section{Mancang Zhao}

Department of Oncology

General Hospital of Beijing Command of PLA

No. 28 Fuxing Road Haidian District

Beijing 100700, China

e-mail: jzwlcn@126.com

Submitted: 15.01 .2013

Accepted: $\quad 29.08 .2013$ 